\title{
Facial rejuvenation for middle-aged women: a combined approach with minimally invasive procedures
}

REVIEW

\author{
Alberto Goldman' \\ Uwe Wollina ${ }^{2}$ \\ 'Clinica Goldman, Porto Alegre, RS, \\ Brazil; ${ }^{2}$ Department of Dermatology \\ and Allergology, Hospital Dresden- \\ Friedrichstadt, Academic Teaching \\ Hospital of the Technical University \\ of Dresden, Dresden, Germany
}

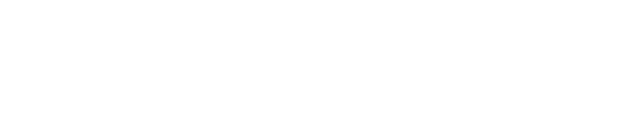

\begin{abstract}
Facial rejuvenation is a significant process involved in restoring youthfulness. The introduction of less invasive procedures has increased acceptance of such procedures. Often a combination of different techniques allows individualized treatment with optimal outcomes. Furthermore, this leads to a natural look without a significant downtime. We report herein the use of such a combined approach in middle-aged women with particular emphasis on botulinum toxin type A, dermal fillers, and chemical peels.
\end{abstract}

Keywords: facial rejuvenation, middle-aged females, botulinum toxin, fillers, chemical peels

In the past the concept of facial rejuvenation was dominated by invasive surgical procedures, in particular different techniques of partial and full face lift. Nowadays there is a clear tendency for less invasive procedures which are quicker and simpler and that do not divert patients from their daily routine and activities for prolonged periods. They can provide effective, safe, relatively long-lasting, and natural results. ${ }^{1,2}$

With the development of new technologies and techniques, greater knowledge of tissue anatomy and physiology, stem cells, lasers and other energy sources, new fillers, and the ever more versatile use of botulinum toxin type A (BTX-A), a new perspective in cervicofacial rejuvenation treatments has been established. In the following we will describe our practice and experiences. We believe that needs for rejuvenation and the appropriate choice of the medical armamentarium are age- and gender-dependent so we focused on women aged 30 to 50 years. ${ }^{3}$

\section{Etiological factors related to skin aging}

The aging process is related to intrinsic and extrinsic factors. The main frequent changes observed among women aged between 30 and 60 years due to the natural and continuing aging process are the appearance of small wrinkles, atrophy of subcutaneous fat, and decreases in collagen and elastin production. There is also an evident decrease in the turnover of skin cells. Gravidity and muscular aging are involved in more evident folds, increasing skin flaccidity. Repeated contractions of the mimic muscles might be involved in the process. In young participants an magnetic resonance imaging (MRI) study was used to demonstrate that the anterior convexity curve of the mimic muscles is due to the existence of a deep fat pad at the back of these muscles. While this mass of fat is effectively a mechanical sliding plane, its convexity constitutes the founding principle of a youthful midface appearance. With age, the muscle gradually straightens and shortens,
Correspondence: Alberto Goldman

Clinica Goldman, Av. Augusto Meyer

I63 conj. I203, 90550-II0

Porto Alegre, RS, Brazil

Email alberto@goldman.com.br 
due to the repeated contractions that expel the underlying fat and increase the tonus at rest. The structural aging becomes visible and, with time, the person's expression consequently becomes more rigid. ${ }^{2}$ The skin becomes drier and thinner and there is an evident loss in the skin texture and elasticity. A variety of external or extrinsic factors often act together with the aging process to prematurely age the skin. The most important and aggressive extrinsic factor related to aging is caused by sun exposure (photoaging). Other external factors include repetitive or exaggerated mimic expressions, gravity, sleeping position, and smoking. The combination of extrinsic and intrinsic aging factors determine different aspects and characteristics in patients aged under 50 years. The most frequent are skin and deep structure flaccidity, wrinkle expression in the upper third of the face, tear through, increase in the nasolabial fold, drop of the angle of the mouth, loss of definition in the mandibular border, cervico-facial platysmal bands, changes in skin pigmentation, and evident veins (or telangiectasia). ${ }^{1}$

Since symmetry is one of the major biologic factors contributing to facial attractiveness, facial rejuvenation procedures are commonly aimed at improving symmetry. There is a need for adapting and specifying esthetic approaches to the special needs of the individual patient.

We will now discuss three minimally invasive procedures; smoothing wrinkles and folds by temporary chemodenervation of facial muscles with botulinum toxin, correction of volume loss by fillers, and improvement of skin surface and dyschromia by peels.

\section{Botulinum toxin type A (BTX-A)}

BTX-A is produced by Clostridium botulinum. Synthesized as a single-chain polypeptide of $\sim 150 \mathrm{kDa}$, BTX has relatively little potency until it is cleaved by trypsin or bacterial enzymes into two chains, a heavy chain of $100 \mathrm{kDa}$ responsible for binding to the target structure and a light chain of $50 \mathrm{kDa}$ known as the toxifying chain. These two chains are linked together by a disulfide bond. The light chain contains a $\mathrm{Zn}^{2+}$-binding motif with enzymatic activity. BTX-A is a zinc-dependent endopeptidase.

When BTX is injected into a muscle, the heavy chain of the molecule binds to glycoproteins expressed specifically on cholinergic nerve endings. After internalization of the whole molecule by pinocytosis, the chains are cleaved within the cytoplasm. The light chain binds with high specificity to the soluble N-ethylmaleimide-sensitive fusion (SNARE) attachment protein receptors essential for exocytosis of acetylcholine. BTX-A cleaves plasma membrane synaptosome associated protein [SNAP-25] that results in a blockade of vesicle fusion. ${ }^{4,5}$
Two to five days after BTX is injected into a muscle paresis occurs which lasts for at least three months and gradually starts to wear off. The subjective duration of action in a given patient seems to be stable, but there is a great variability between different people. Dose-effect correlations are known for electromyographic activity. Even very low doses produce some effect. By increasing the doses a plateau is reached where further dose increases will not result in stronger effects. Dose-duration correlations exist to a lesser extent. Saturation will be seen with higher dosages after approximately three months. ${ }^{5}$

On the European Market, three different branches of BTX-A are officially registered: Botox $^{\circledR} /$ Vistabel $^{\circledR}$ (Allergan), Dysport ${ }^{\circledR}$ (Ipsen)/Azzalure ${ }^{\circledR}$ (Galderma), and Xeomin $^{\circledR} /$ Bocouture $^{\circledR}$ (Merz). The units are not directly comparable. The products have different amounts of complexing proteins or are free of complexing proteins (ie, Xeomin ${ }^{\circledR} /$ Bocouture $\left.^{\circledR}\right){ }^{6}$ The diffusion of the different drugs seems to be dependent on concentration. ${ }^{7}$ BTX-A has an excellent safety profile ${ }^{8}$ and has been used extensively for facial rejuvenation with a focus on hyperkinetic wrinkles and to improve facial wound healing after surgery. ${ }^{9-12}$

\section{Fillers}

Fillers can be permanent or temporary. Different products can be inserted under the skin in different layers to correct depressed folds, wrinkles, furrows, scars, angles, and flaccidity. The main concept is to create a volume or even to replace an original volume compromise for the aging process of the face and neck.

The types and number of dermal fillers have evolved, allowing clinicians to select the most appropriate agent for each specific purpose. Filler properties differ both between and among classes, so clinicians must have a thorough understanding of these properties and the best techniques to use to provide the most satisfactory outcomes.

Fillers can be divided into permanent and temporary categories. The latter are metabolized by the human body. Permanent fillers are no longer recommended because their long term safety has not been established. Nevertheless, some authors have published excellent results for the use of permanent fillers like ArteFill ${ }^{\circledR}{ }^{13}$

Temporary fillers consist of various substances including collagen, hyaluronic acid (HA), alginates, and other polysaccharides. Substances such as polylactic acid or hydroxylapatite are considered to be semipermanent fillers with a longer half life. ${ }^{14}$ 
The most common used substance today is HA, a natural polysaccharide and a component of human dermis and epidermis. HA-based fillers offer excellent biocompatibility while providing the same structural and mechanical properties of normal subcutaneous tissue. Naturally occurring hyaluronan is rapidly broken down by hyaluronidase with a half-life of about 12 hours and eliminated through the lymphatics and by the hepatic metabolism to carbon dioxide and water. ${ }^{14,15}$ Cross-linking gives HA fillers an in vivo life span of 6 to 18 months. The use of HA is particularly attractive for soft-tissue augmentation, because it is hydrophilic and a normal extracellular component of skin. It is directly involved in extracellular matrix function and because of its tolerability profile, it can be used without skin testing. ${ }^{15}$ Corrections of displacement can easily be done by injection of hyaluronidase. ${ }^{16}$

Factors that impact HA persistence include HA concentration, percentage of cross-linkage, type of cross-linkage, water binding capacity, and injection technique. Monophasic gels seem to be more stable compared with diphasic gels. To improve longevity, manufacturers use various agents to cross-link the HA. As a result, the final proportion of cross-linked HA and the degree of cross-linking impact the physical characteristic of the final product. The water-binding capacity - or hydrophilic nature - allows the HA to create larger volumes relative to their mass. Recent study results suggest that increased concentrations of HA prolong persistence. If all other factors are the same among HAs, the product with the highest degree of cross-linking experiences the least amount of degradation by enzymes and free radicals. The cross-linking agents also have an impact on connective tissue reaction to injectable fillers. ${ }^{17}$ Injection technique can play a role in longevity of the dermal filler. Injection into the deep dermis has been shown to increase de novo collagen synthesis, hypothesized to be the result of fibroblast stretching. Therefore as the HA is degraded, novel collagen synthesis replaces the HA which results in longer-lasting correction. Semipermanent fillers often cause more pain during injection. Nerve blocks are used to reduce this during the procedure. ${ }^{18}$ The combination of HA filler with BTX-A has been shown to produce improved and longer lasting effects for glabellar lines than either BTX-A or filler alone. ${ }^{19}$

Another filler with potential long term effects is autologous adipose tissue used for facial sculpturing. Adipose tissue is believed to constitute an ideal source of uncultured stromal stem cells. By optimizing the harvesting, storage, and transplantation of adipose tissue, long-lasting results can be obtained. ${ }^{20}$ The long lasting volumizing in many patients seems to be due to mesenchymal stem cells that eventually differentiate into fibroblasts, adipocytes, and blood vessel. ${ }^{21-23}$ Autologous fat transfer has been successfully used for facial lipodystrophy and for facial sculpturing in esthetic facial surgery. ${ }^{24,25}$ Autologous fat transfer to the midface has definite long-term volume augmentation results. On average, approximately $32 \%$ of the injected volume remains at 16 months. However, some variability exists in the percentage of volume that remains, meaning that a touch-up procedure may be required. ${ }^{26}$

In the future is it hoped that better identification of and enrichment with stem cells for adipose transfer will create a more predictable long-term outcome. ${ }^{27}$ Progenitor, endothelial, and mesenchymal stem cells derived from adipose tissue could therefore not only be central to plastic and reconstructive surgery applications, but also become the focus of an array of therapeutic solutions for many disease conditions, such as those affecting bone, cartilage, muscle, liver, kidney, the heart, the brain, and the pancreas, expanding the possible indications and translational potential of tissue, cell-based, and regenerative medicine strategies. ${ }^{21}$

\section{Chemical peeling}

Chemical peeling is the topical application of chemical agents to the skin, which causes controlled destruction of part of or the entire epidermis, with or without the dermis, leading to exfoliation and removal of superficial lesions, followed by regeneration of new epidermal and dermal tissues. Indications for chemical peeling include pigmentary disorders, superficial acne scars, aging skin changes, and benign epidermal growths. Peels have been classified according to the chemical compounds involved or the depth of the exfoliation. ${ }^{28}$

Superficial chemical peels are currently performed with various compounds including trichloracetic acid, alpha hydroxy acids, and Jessner's solution (14\% lactic acid, $14 \%$ resorcinol, and 14\% salicylic acid). Alpha hydroxy acids, in particular glycolic acid (GA), are common chemical peel agents. GA peels in concentration ranging from $20 \%-70 \%$ have been shown to be effective in reducing facial hyperpigmentation and fine lines/wrinkles. ${ }^{29}$

Based on the available information, these salicylic ingredients are safe for use when formulated to avoid skin irritation or to avoid increasing the skin's sun sensitivity, or, when increased sun sensitivity would be expected, directions for use include the daily use of sun protection. ${ }^{30,31}$

Kligman and Kligman have developed salicylic acid, a beta hydroxy acid (30\% in a hydro ethanolic vehicle), as an 
alternative peel to GA. ${ }^{32}$ The benefits of salicylic acid peels are resurfacing moderately photodamaged facial skin, fading of pigment spots, decreased surface roughness, and reduction of fine lines also in pigmented skin..$^{33}$

Derivatives of salicylic acid, especially long-chain fatty acylconjugates of salicylic acid, have been claimed to be more effective at lower concentrations for skin peeling use. A lipophilized ester-derivative of salicylic acid, capryloyl salicylic acid (CSA), has been shown to be an effective exfoliating agent due to its reduced penetration in the skin. ${ }^{34}$

Salicylic acid causes a loss of cornified cells from the horny layer followed by the activation of the epidermal basal cells and the underlying fibroblasts. ${ }^{35}$ In photoexposed human facial skin, immature cornified envelopes (CEs) were replaced with mature CEs four weeks after treatment with salicylic-polyethylene glycol (PEG). Restoration of photodamaged stratum corneum by this treatment involved the normalization of keratinocyte differentiation. ${ }^{36}$

\section{Our concept of minimally invasive facial rejuvenation in women between 30 and 50 years of age}

There is no doubt that that classic surgical and nonsurgical procedures such as blepharoplasty, rhytidoplasty, or laser peelings still represent important techniques in esthetic and facial rejuvenation. It is also obvious that the combination of these traditional treatments with other procedures that offer improved skin quality and texture, wrinkles, low risk, good cost-effectiveness, low morbidity, and quick recovery represent an important advance and a real tendency. In this context, BTX-A injection combined with different types of fillers or soft tissue augmentation represent an important nonsurgical resource that, combined with traditional techniques, can improve or extend the results.

These two procedures, BTX and fillers, can also be combined with some kinds of lasers or light sources in order to produce a nonablative or fractional skin rejuvenation, superficial improvement, pigmented lesions, vascular alterations, wrinkles, and acne scars as well as to offer adequate skin tightening. The concept is to treat the wrinkles and folds and also improve the photodamage related to the aging process and skin laxity.

This report is based on extensive experience with combined procedures in facial rejuvenation. We will focus on the period of time from July 1996 to June 2010. A total of 1,559 patients ( $91 \%$ female) have been treated, aged under 50 years. Middle-aged women are a major group for esthetic treatments. Fifty point three percent of patients received a
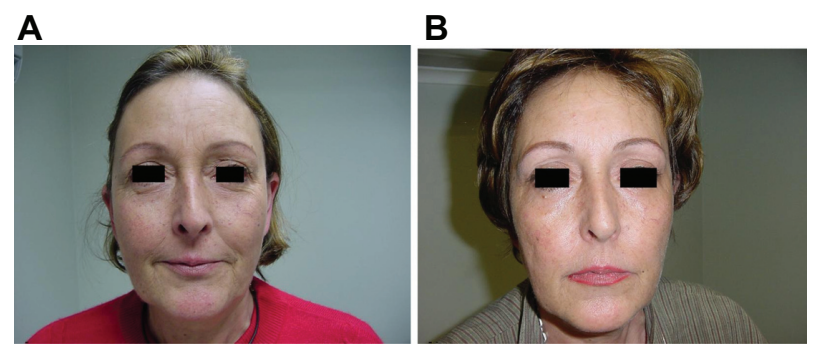

Figures IA and IB A 60 year-old patient after hyaluronic acid injection in nasolabial fold, malar region, and mouth commissure $(1.6 \mathrm{~mL})$ and Intense Pulsed Light rejuvenation. Result 2 months after HA injection and two sessions of IPL and Nd:YAG laser (Restylane, Perlane, and Photosilk Plus and Synchro - Deka, Italy).

combined treatment. The major combination was BTX-A and fillers ( $\mathrm{n}=784$, with $97 \%$ females). The main treated areas were periorbital, frontal, glabellar, platysma, depressor anguli oris, and nasal (Figures 1-7). Eighty-four percent of these patients received a reinjection of BTX-A on average five months after the first treatment. This high number indicates patients' satisfaction leading to a continuation of the initial procedures despite costs.

Facial plastic surgery procedures, such as cervicofacial rhytidoplasty or blepharoplasty, are the most common procedures used in combination with BTX-A and fillers. Whether the optimal moment for injecting BTX-A is before or after the surgery remains a subject of debate. A potential advantage of using it prior to surgical intervention is the stabilization of cutaneous or even myocutaneous tissues. This, in turn, would facilitate their adequate accommodation, decreasing tension in the incision, suture, flaps and traction areas, thus improving the quality of the cicatricial process. Despite its transient duration and relatively high cost limit the use of neurotoxins as an adjunctive agent in surgery is suitable for most patients. In the first weeks following surgery, the relaxing effect of BTX-A is disguised by tissue edema. Thus, its useful life and cosmetic results and effects are considerably shortened. The versatility and reproductive effect of BTX-A among these patients represent an important characteristic of this approach.

The aspect of a frontal surgical surgery (open or videoassisted) can be temporarily mimicked or reproduced with an

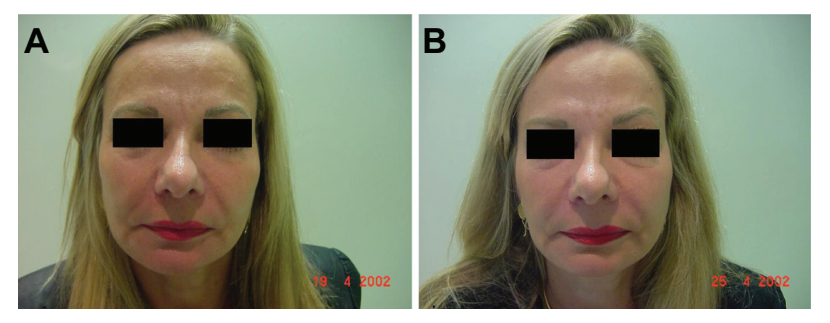

Figures 2A and 2B A 53-year-old female patient treated with $1.8 \mathrm{~mL}$ of $\mathrm{HA}$ (Juvederm) in the mandibular border, malar region, mouth commissure (marionette lines), and nasolabial fold. 
A

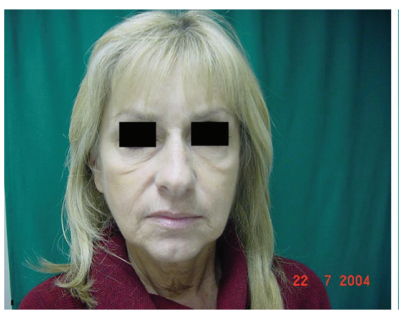

B

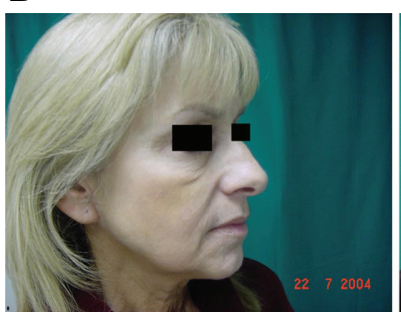

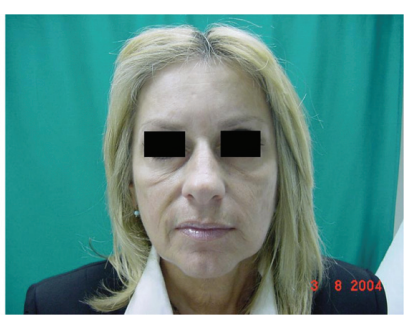

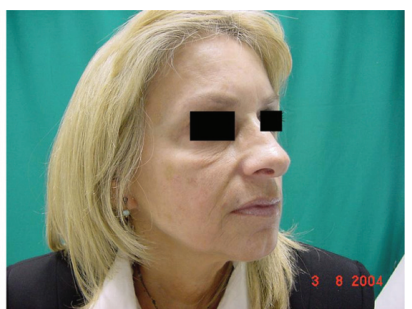

Figures 3A and 3B A 5I-year-old patient. HA injections in the wrinkles around the mouth, mandibular border, malar region, mouth commissure, and nasolabial fold. Total volume injection was $1.9 \mathrm{~mL}$.

adequate application into the frontal, corrugators, procerus, and orbicularis oculi muscles. This resource is useful for patients aged under 50 years, who do not want or do not have clinical conditions to undergo surgery or prefer a less invasive procedure. BTX-A application can effectively offer an elevation of the lateral aspect of the eyebrow or of the mouth commissure. Thus, for the treatment of eyelid ptosis, whatever technique is used (open, endoscopic, or combined approach), previous neurotoxin application may be very useful for inhibiting or smoothing possible cutaneous and muscular creases in the treated areas. On the upper third of the face, BTX-A can attenuate the hyperdynamic wrinkles caused by muscular activity, eliminating the need for either a coronal incision in treating the frontal musculature or for an endoscopic procedure. When it is injected into the palpebral region, it is possible to obtain an elevation of the caudal portion of the eyebrow and modify its shape or position, which is useful especially when performing a blepharoplasty. This palpebral elevation (lifting effect) brings about significant

A

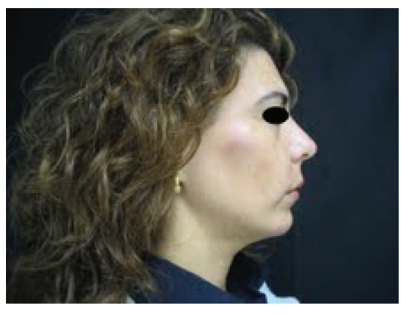

B

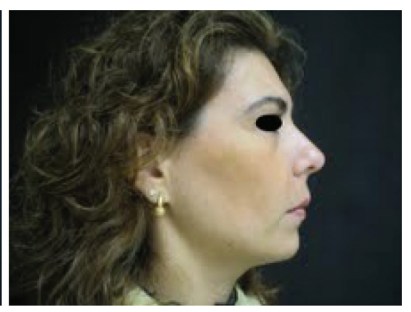

Figure 4 A 48-year-old woman. BTX-A in the upper third of the face. HA filler for mouth commisure, vermillion, malar region, and mandibular border ( $1.6 \mathrm{ml})$; A) before and $\mathbf{B}$ ) after the procedure.
A

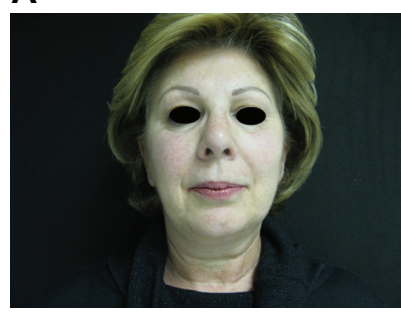

B

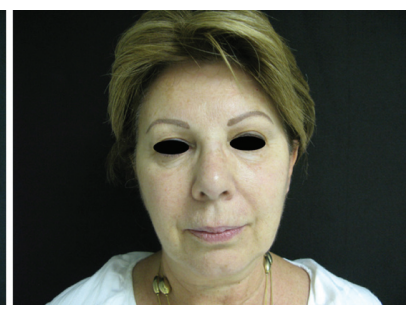

Figure 5 A 52-year-old woman. A) Before and B) after full face trichloracetic acid (TCA) peel, BTX-A for the depressor anguli oris and the upper third of the face. $1.2 \mathrm{ml}$ of HA filler was injected in both the nasolabial fold and oral commissure.

appearance of rejuvenation as well as the improvement of "crow's feet". By using this combination, it is possible, in selected cases, to decrease the quantity of skin to be removed and consequently, to decrease the scar dimension. Although many researchers promote its postoperative use, there is no consensus as to the ideal time of application in these cases.

BTX-A injection into the lateral and anterior cervical regions and in the depressor anguli oris is capable of attenuating the platysmal band and elevating the angles of the mouth, creating harmony between this area and the rest of the face and improving the result of the rhytidoplasty. Although BTX-A can be used in combination with fillers, lasers, and surgical procedures or even applied into other areas of the face and neck, we should use common sense and carefully examine the patient's anatomy when using this neurotoxin in order to avoid excessive muscle relaxation, muscle paralysis, or the end result of a face incapable of natural expressions.

\section{Combination with peels}

Several fillers, skin care techniques, chemical peelings, dermabrasion, and different types of ablative and nonablative lasers and energy sources can be combined with BTX-A use in order to prevent or to treat the aging process. To improve superficial skin texture and pigmentary problems chemical peels have been used successfully.

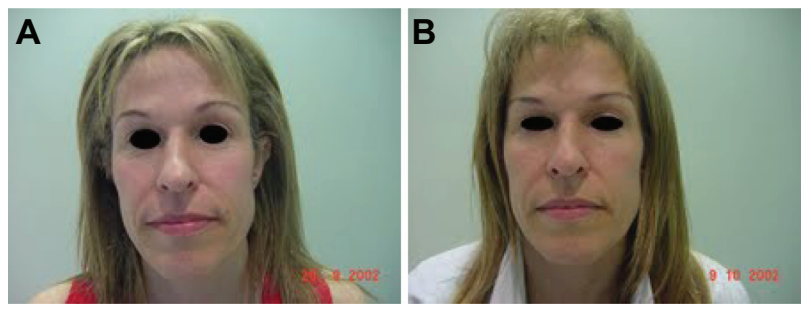

Figure 6 A 50-year-old woman. A) Before treatment and B) She was treated by a combination of HA filler in the vermillion, nasolabial folds, and oral commissure. 


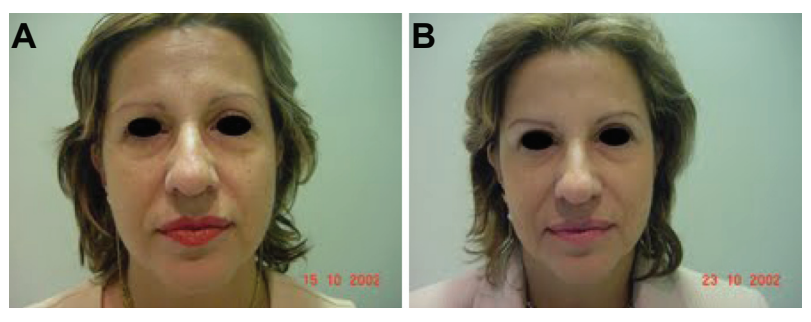

Figure 7 A 52-year-old woman. A) Before and B) after treatment with TCA peel, HA filler for nasolabials, and BTX-A in the upper third of the face.

Twenty patients ( 15 females and 5 males, skin type I-IV) with acne, acne scars, or actinic damage were included in an open clinical trial. All of them underwent a preparation period of two weeks before first peeling with 5\% CSA solution. The peeling was performed twice a month for a total of 4 to 5 treatments. The concentration of CSA was increased to $10 \%$ by the second treatment. Patients' global assessments and physicians' global assessments of the CSA peels were considered good or better in its clinical effects in 18 of 18 and 17 of 18 patients who completed the series of peels. Two patients dropped out because of intolerance to either the preparation agents or to $5 \% \mathrm{CSA}$. In all other patients the treatment was well tolerated without any serious adverse effects. All patients reported some tingling sensations and desquamation $24-48$ hours after the procedure. There was no downtime from work. There is some evidence that smoking might negatively affect the efficacy of CSA peels. CSA peels are well tolerated in most patients of skin type I-IV with improvement of inflammatory acne lesions, facial acne scars, radiance, pigmentary changes, and fine wrinkling. ${ }^{37}$ Future researchers could investigate the performance of repeated series every other year.

\section{Perspectives}

Improved knowledge of cellular and skin aging, the use of stem cells, new and safer filler options, the technical evolution of surgical techniques, advances of laser sources, fractional laser and other energy sources, as well as new neurotoxin complexes represents a promising future in the treatment of the aging aspects of patients aged 30 to 50 years.

\section{Conclusions}

The appropriate evaluation and correct use of these minimally or less invasive techniques led to a younger and healthier look for the treatment of facial aging. BTX-A is one of the most commonly used nonsurgical techniques. This neurotoxin in conjuction with fillers has a low risk, efficiently treats skin aging, and also prevents or reduces some aspects and characteristics related to the facial aging process. Chemical peels like CSA can further contribute to a youthful facial appearance with a more even skin tone and increased radiance.

\section{Disclosure}

The authors report no conflicts of interest in this work.

\section{References}

1. Beer K, Beer J. Overview of facial aging. Facial Plast Surg. 2009;25(5): 281-284.

2. Le Louarn C. Muscular aging and its involvement in facial ageing: the face recurved concept (in French). Ann Dermatol Venereol. 2009;136(Suppl 4):S67-S72.

3. Wollina U, Payne CR. Aging well - the role of minimally invasive aesthetic dermatological procedures in women over 65. J Cosmet Dermatol. 2010;9(1):50-58.

4. Breidenbach MA, Brunger AT. New insights into clostridial neurotoxinSNARE interactions. Trends Mol Med. 2005;11(8):377-381.

5. Wollina U. Botulinum toxin: non-cosmetic indications and possible mechanisms of action. J Cutan Aesth Surg. 2008;1(1):3-6.

6. Carruthers A, Carruthers J. Botulinum toxin products overview. Skin Therapy Lett. 2008;13(6):1-4.

7. Pickett A, Dodd S, Rzany B. Confusion about diffusion and the art of misinterpreting data when comparing different botulinum toxins used in aesthetic applications. J Cosmet Laser Ther. 2008;10(3):181-183.

8. Wollina U, Konrad H. Managing adverse events associated with botulinum toxin A: a focus on cosmetic procedures. Am J Clin Dermatol. 2005;6(3):141-150.

9. Ogden S, Griffiths TW. A review of minimally invasive cosmetic procedures. Br J Dermatol. 2008;159(5):1036-1050.

10. Carruthers J, Carruthers A. Botulinum toxin in facial rejuvenation: an update. Dermatol Clin. 2009;27(4):417-425.

11. Gadhia K, Walmsley AD. Facial aesthetics: is botulinum toxin treatment effective and safe? A systematic review of randomised controlled trials. Br Dent J. 2009;207(5):E9.

12. Gassner HG, Brissett AE, Otley CC, et al. Botulinum toxin to improve facial wound healing: a prospective, blinded, placebo-controlled study. Mayo Clin Proc. 2006;81(8):1023-1028.

13. Lemperle G, Knapp TR, Sadick NS, Lemperle SM. ArteFill permanent injectable for soft tissue augmentation: I. Mechanism of action and injection techniques. Aesthetic Plast Surg. 2010;34(3):264-272.

14. Beer K, Lupo MP. Making the right choices: attaining predictable aesthetic results with dermal fillers. J Drugs Dermatol. 2010;9(5): $458-465$.

15. Carruthers J, Cohen SR, Joseph JH, Narins RS, Rubin M. The science and art of dermal fillers for soft-tissue augmentation. J Drugs Dermatol. 2009;8(4):335-350.

16. Rzany B, Becker-Wegerich P, Bachmann F, et al. Hyaluronidase in the correction of hyaluronic acid-based fillers: a review and a recommendation for use. J Cosmet Dermatol. 2009;8(4):317-323.

17. Yeom J, Bhang SH, Kim BS, et al. Effect of cross-linking reagents for hyaluronic acid hydrogel dermal fillers on tissue augmentation and regeneration. Bioconjug Chem. 2010;21(2):240-247.

18. Marmur E, Green L, Busso M. Controlled, randomized study of pain levels in subjects treated with calcium hydroxylapatite premixed with lidocaine for correction of nasolabial folds. Dermatol Surg. 2010;36(3):309-315.

19. Carruthers J, Carruthers A. A prospective, randomized, parallel group study analyzing the effect of BTX-A (Botox) and nonanimal sourced hyaluronic acid (NASHA, Restylane) in combination compared with NASHA (Restylane) alone in severe glabellar rhytides in adult female subjects: treatment of severe glabellar rhytides with a hyaluronic acid derivative compared with the derivative and BTX-A. Dermatol Surg. 2003;29(8):802-809. 
20. Gimble J, Guilak F. Adipose-derived adult stem cells: isolation, characterization, and differentiation potential. Cytotherapy. 2003;5(5):362-369.

21. Tremolada C, Palmieri G, Ricordi C. Adipocyte transplantation and stem cells: plastic surgery meets regenerative medicine. Cell Transplant. 2010 [Epub ahead of print].

22. Faustini M, Bucco M, Chlapanidas T, et al. Nonexpanded mesenchymal stem cells for regenerative medicine: yield in stromal vascular fraction from adipose tissues. Tissue Eng Part C Methods. 2010 [Epub ahead of print].

23. Marra K, Rubin JP. Adipose stem cells for soft tissue regeneration. Handchir Mikrochir Plast Chir. 2010;42(2):124-128.

24. Cohen G, Treherne A. Treatment of facial lipoatrophy via autologous fat transfer. J Drugs Dermatol. 2009;8(5):486-489.

25. Wollina U, Goldman A, Berger U, Abdel-Naser MB. Esthetic and cosmetic dermatology. Dermatol Ther. 2008;21(2):118-130.

26. Meier JD, Glasgold RA, Glasgold MJ. Autologous fat grafting: longterm evidence of its efficacy in midfacial rejuvenation. Arch Facial Plast Surg. 2009;11(1):24-28.

27. Mosna F, Sensebé L, Krampera M. Human bone-marrow and adipose tissue mesenchymal stem cells: a user's guide. Stem Cells Dev. 2010 [Epub ahead of print].

28. Zakopoulou N, Kontochristopoulos G. Superficial chemical peels $J$ Cosmet Dermatol. 2006;5(3):246-253.

29. Grover C, Reddu BS. The therapeutic value of glycolic acid peels in dermatology. Indian J Dermatol Venereol Leprol. 2003;69(2):148-150.
30. Fung W, Orak D, Re TA, Haughey DB. Relative bioavailability of salicylic acid following dermal application of a 30\% salicylic acid skin peel preparation. J Pharm Sci. 2008;97(3):1325-1328.

31. Cassano N, Alessandrini G, Mastrolonardo M, Vena GA. Peeling agents: toxicological and allergological aspects. J Eur Acad Dermatol Venereol. 1999;13(1):14-23.

32. Kligman D, Kligman AM. Salicylic acid peels for the treatment of photoaging. Dermatol Surg. 1998;24(2):325-328.

33. Bari AU, Iqbal Z, Rahman SB. Tolerance and safety of superficial chemical peeling with salicylic acid in various facial dermatoses. Indian J Dermatol Venereol Leprol. 2005;71(2):87-90.

34. Saint-Léger D, Lévêque JL, Verschoore M. The use of hydroxy acids on the skin: characteristics of C8-lipohydroxy acid. J Cosmet Dermatol. 2007;6(1):59-65.

35. Imayama S, Ueda S, Isoda M. Histologic changes in the skin of hairless mice following peeling with salicylic acid. Arch Dermatol. 2000;136(11):1390-1395.

36. Dainichi T, Ueda S, Furue M, Hashimoto T. By the grace of peeling: the brace function of the stratum corneum in the protection from photo-induced keratinocyte carcinogenesis. Arch Dermatol Res. 2008;300(Suppl 1):S31-S38.

37. Wollina U. Capryloyl salicylic acid (CSA) peel for acne and actinic damage. Kosmet Med. 2009;30(4):142-145.
Clinical Interventions in Aging

\section{Publish your work in this journal}

Clinical Interventions in Aging is an international, peer-reviewed journal focusing on evidence-based reports on the value or lack thereof of treatments intended to prevent or delay the onset of maladaptive correlates of aging in human beings. This journal is indexed on PubMed Central, MedLine, the American Chemical Society's 'Chemical

\section{Dovepress}

Abstracts Service' (CAS), Scopus and the Elsevier Bibliographic databases. The manuscript management system is completely online and includes a very quick and fair peer-review system, which is all easy to use. Visit http://www.dovepress.com/testimonials.php to read real quotes from published authors. 